\title{
Probe interval graphs and probe unit interval graphs on superclasses of cographs
}

$$
\begin{aligned}
& \text { Flavia Bonomo }\left.^{1,2}\right|^{\mid} \\
& \text {Luciano N. Grippo }
\end{aligned}
$$

\author{
Guillermo Durán $1,3, ⿲^{1}$
Martín D. Safe
}

${ }^{1}$ CONICET, Argentina

${ }^{2}$ Depto. de Computación, FCEyN, Universidad de Buenos Aires, Buenos Aires, Argentina

${ }^{3}$ Depto. de Matemática and Instituto de Cálculo, FCEyN, Universidad de Buenos Aires, Buenos Aires, Argentina

${ }^{4}$ Depto. de Ingeniería Industrial, FCFM, Universidad de Chile, Santiago, Chile

${ }^{5}$ Instituto de Ciencias, Universidad Nacional de General Sarmiento, Los Polvorines, Argentina

received $26^{\text {th }}$ March 2012, revised $8^{\text {th }}$ July 2013, accepted $1^{\text {st }}$ August 2013.

\begin{abstract}
A graph is probe (unit) interval if its vertices can be partitioned into two sets: a set of probe vertices and a set of nonprobe vertices, so that the set of nonprobe vertices is a stable set and it is possible to obtain a (unit) interval graph by adding edges with both endpoints in the set of nonprobe vertices. Probe (unit) interval graphs form a superclass of (unit) interval graphs. Probe interval graphs were introduced by Zhang for an application concerning the physical mapping of DNA in the human genome project. The main results of this article are minimal forbidden induced subgraphs characterizations of probe interval and probe unit interval graphs within two superclasses of cographs: $P_{4}$-tidy graphs and tree-cographs. Furthermore, we introduce the concept of graphs class with a companion which allows to describe all the minimally non-(probe $\mathcal{G}$ ) graphs with disconnected complement for every graph class $\mathcal{G}$ with a companion.
\end{abstract}

Keywords: $P_{4}$-tidy graphs, forbidden induced subgraphs, probe interval graphs, probe unit interval graphs, treecographs

\footnotetext{
${ }^{\dagger}$ Email: fbonomo@dc.uba.ar. Partially supported by ANPCyT PICT 2012-1324, UBACyT Grant 20020100100980, and CONICET PIP 112-200901-00178 (Argentina).

${ }^{\ddagger}$ Email: gduran@dm.uba.ar. Partially supported by FONDECyT Grant 1110797 and Millennium Science Institute "Complex Engineering Systems" (Chile), and ANPCyT PICT 2012-1324, UBACyT Grant 20020100100980, and CONICET PIP 112200901-00178 (Argentina)

§Email: Igrippo@ungs .edu . ar. Partially supported by ANPCyT PICT 2012-1324, UBACyT Grant 20020100100980, and CONICET PIP 112-200901-00178 (Argentina)

IEmail: msafe@ungs .edu. ar. Partially supported by ANPCyT PICT 2012-1324, UBACyT Grant 20020100100980, and CONICET PIP 112-200901-00178 (Argentina)
}

1365-8050 @ 2013 Discrete Mathematics and Theoretical Computer Science (DMTCS), Nancy, France 


\section{Introduction}

A graph $G$ is an interval graph if it is the intersection graph of a family of open intervals in the real line; i.e., there is a one-to-one correspondence between the vertex set of $G$ and a collection $\mathcal{S}$ of intervals in the real line such that two vertices of $G$ are adjacent if their corresponding intervals intersect. If so, $\mathcal{S}$ is called an interval model of $G$. A unit interval graph is an interval graph admitting an interval model with all its intervals having the same length. Interval and unit interval graphs have well-known characterizations by minimal forbidden induced subgraphs [15, 18]. A graph is probe (unit) interval if its vertices can be partitioned into two sets: a set of probe vertices and a set of nonprobe vertices, so that the set of nonprobe vertices is a stable set and it is possible to obtain a (unit) interval graph by adding edges with both endpoints in the set of nonprobe vertices.

Probe interval graphs form a superclass of interval graphs and were introduced by Zhang [23] as a research tool in the frame of the human genome project. An open question from a combinatorial viewpoint is that of characterizing the classes of probe interval and probe unit interval graphs by forbidden induced subgraphs. Partial results in this direction were obtained in the following articles. Brown and Lundgren [2] prove that the class of bipartite probe interval graphs and the class of complements of 2-clique circulararc graphs are each equivalent to the class of point interval bigraphs. In the same article the authors characterize those probe bipartite graphs such that the partition into probe and nonprobe vertices coincides with the bipartition (as bipartite graphs) but the problem of characterizing bipartite probe interval graphs remains open. However, they present a conjecture describing the complete family of forbidden induced subgraphs for the class of bipartite probe interval graphs. Sheng [19] characterizes probe interval graphs by forbidden induced subgraphs within the class of cycle-free graphs. Brown, Lundgren, and Sheng [3] do the same work for the class of probe unit interval graphs. Pržulj and Corneil [17] study the class of probe interval graphs within the class of 2-trees, finding a wide list of forbidden induced subgraphs which belong to this class.

The main results of this paper are characterizations of probe interval graph and probe unit interval graphs by a set of minimal forbidden induced subgraphs within two superclasses of cographs: treecographs and $P_{4}$-tidy graphs. Both superclasses of cographs are defined recursively from the disjoint union and the join operation. The study of minimal forbidden induced subgraphs of probe interval graphs and probe unit interval graphs within these classes in connection with the join operation gives rise to introduce the concept of companion of a graph class (cf. Section 5). The results on probe unit interval graphs presented in this article follow from the connection between the behavior of the join operation and the companion class of unit interval graphs. The characterizations of probe interval and probe unit interval graphs within the class of tree-cographs are also based on the characterizations presented in [19] and [3] respectively, as well as on Theorem 10 , which characterizes probe interval graphs and probe unit interval within the class of co-bipartite graphs. The last result follows from results on domination in graphs presented in [4] leads to characterizations of probe interval graphs and probe unit interval graphs within the class of complements of trees.

The paper is organized as follows. In Section 2, we introduce some terminology and state some results used throughout the article. Section 3 is devoted to characterize by forbidden induced subgraphs probe interval graphs and probe unit interval graphs within co-bipartite graphs. In Section 4 , we present characterizations by forbidden induced subgraphs within two superclasses of cographs, namely tree-cographs and $P_{4}$-tidy graphs. The results given in Section 4 can be proved using a theorem presented in Section 5 . Nevertheless, we decided to show an alternative proof that implicitly uses this theorem, for the 
convenience of the reader. In Section 5 the concept of hereditary class with a companion is introduced. Theorem 22 is the main result of Section 5. Mostly, the characterizations of Section 7 are based on Theorem 22. In Section 6 characterizations for probe $\left\{3 K_{1}, C_{4}, C_{5}\right\}$-free graphs are presented. The class of $\left\{3 K_{1}, C_{4}, C_{5}\right\}$-free graphs is the companion of the class of unit interval graphs. Finally, Section 7 is devoted to forbidden induced subgraphs characterizations of probe unit interval graphs within tree-cographs and $P_{4}$-tidy graphs.

\section{Preliminaries}

For concepts and notation not defined here we refer the reader to [22]. All graphs in this article are simple (i.e., without loops or multiple edges). Let $G$ be a graph. The vertex set of $G$ is denoted by $V(G)$ and the edge set by $E(G)$. If $u, v \in V(G)$ and $u v \notin E(G), u v$ is called a nonedge of $G$. For each vertex $v$ of $G$, $N_{G}(v)$ denotes the neighborhood of $v$ in $G$ and $N_{G}[v]$ denotes closed neighborhood $N_{G}(v) \cup\{v\}$. The degree of a vertex $v$, denoted by $d_{G}(v)$, is the number of vertices in $N_{G}(v)$

Given a subset $A \subseteq V(G), G[A]$ stands for the subgraph of $G$ induced by $A$. Two vertices $v$ and $w$ of $G$ are false twins (resp. true twins) if $N_{G}(v)=N_{G}(w)$ (resp. $N_{G}[v]=N_{G}[w]$ ). The complement graph of $G$ is denoted $\bar{G}$. Let $G_{1}$ and $G_{2}$ two graphs. The disjoint union of $G_{1}$ and $G_{2}$ is denoted by $G_{1}+G_{2}$. If $\mathcal{H}$ is a collection of graphs, a graph $G$ is $\mathcal{H}$-free if $G$ contains no induced subgraph isomorphic to some graph belonging to $\mathcal{H}$. Given a graph $H \in \mathcal{H}$, we say that $G$ contains no induced $H$ if $G$ contains no induced subgraph isomorphic to $H$.

Let $\mathcal{G}$ be a family of graphs. A graph belonging to $\mathcal{G}$ is called a $\mathcal{G}$-graph. The family of graphs formed by the complements of the graphs belonging to $\mathcal{G}$ is denoted $c o-\mathcal{G}$. If $G \in \mathcal{G}$ implies that every induced subgraph of $G$ is a $\mathcal{G}$-graph, $\mathcal{G}$ is said to be hereditary. If $\mathcal{G}$ is a hereditary class, a graph $H$ is a minimal forbidden induced subgraph of $\mathcal{G}$, or more briefly, minimally non- $\mathcal{G}$, if $H$ does not belong to $\mathcal{G}$ but every proper induced subgraph of $H$ is a $\mathcal{G}$-graph. Let $(N, P)$ be a partition of $V(G)$ where $N$ is a stable set. A completion of $G$ is a graph $G^{*}$ obtained from $G$ by adding an edge set $F$ whose endpoints belong to the stable set $N$. Let $\mathcal{G}$ be a hereditary class of graphs. $G$ is defined to be probe $\mathcal{G}$ if there exists a partition $(N, P)$ of $V(G)$ and a completion $G^{*}$ of $G$ belonging to $\mathcal{G}$. Under such conditions, $(N, P)$ is called a probe $\mathcal{G}$ partition of $G$, and the vertices belonging to $N$ (resp. $P$ ) are called nonprobe vertices (resp. probe vertices). $\mathcal{P}(\mathcal{G})$ denotes the class of probe $\mathcal{G}$ graphs. Probe classes of graphs have been studied for instance in [1, 4, 5, 6, 9, 14].

The induced path (resp. induced cycle) on $n$ vertices is denoted $P_{n}$ (resp. $C_{n}$ ). The complete graph on $n$ vertices is denoted $K_{n}$ and $n K_{1}$ stands for a stable set on $n$ vertices. Particularly, $C_{3}$ is called triangle. A paw is a triangle with a pendant vertex. A diamond is a four vertex complete graph less an edge. A graph is bipartite if its vertex set can be partitioned into two stable sets. If, in addition, every vertex of one stable set is adjacent to every vertex of the other stable set the graph is defined as complete bipartite. $K_{n, m}$ stands for the complete bipartite graph partitioned into two stable sets with $n$ and $m$ vertices, respectively. For some graphs needed in what follows, see Figure 1 .

Let $A$ be a vertex set that induces $P_{4}$ in $G$. A vertex $v$ of $G$ is said to be a partner of $A$ if $G[A \cup\{v\}]$ contains at least two induced $P_{4}$ 's and $G$ is called $P_{4}$-tidy if each vertex set $A$ inducing a $P_{4}$ in $G$ has at most one partner [8]. The class of $P_{4}$-tidy graphs is an extension of the class of cographs as well as several other classes defined by bounding the number of $P_{4}$ 's according to different criteria; e.g., $P_{4}$ sparse graphs [11], $P_{4}$-lite graphs [12], and $P_{4}$-extendible graphs [13]. A spider [11] is a graph whose vertex set can be partitioned into three sets $S, C$, and $R$, where $S=\left\{s_{1}, \ldots, s_{k}\right\}(k \geq 2)$ is a stable set; 

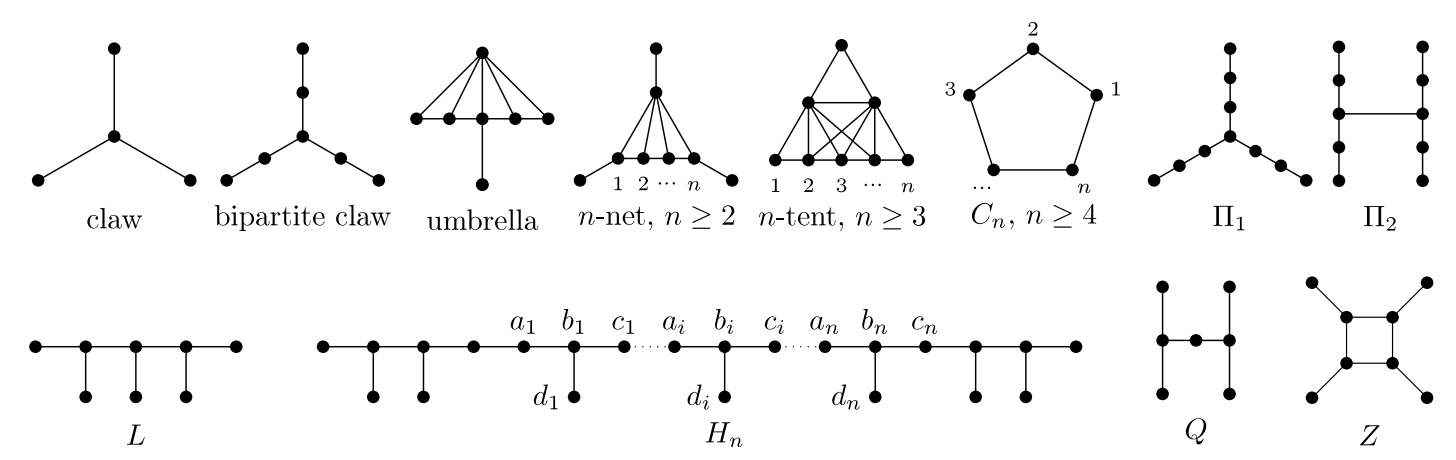

Fig. 1: List of graphs used throughout the paper.

$C=\left\{c_{1}, \ldots, c_{k}\right\}$ is a clique; $s_{i}$ is adjacent to $c_{j}$ if and only if $i=j$ (a thin spider), or $s_{i}$ is adjacent to $c_{j}$ if and only if $i \neq j$ (a thick spider); $R$ is allowed to be empty and if it is not, then all the vertices in $R$ are adjacent to all the vertices in $C$ and nonadjacent to all the vertices in $S$. The triple $(S, C, R)$ is called the spider partition. By $\operatorname{thin}_{k}(H)$ and thick $k_{k}(H)$ we respectively denote the thin spider and the fat spider with $|C|=k$ and $H$ the subgraph induced by $R$. If $R$ is an empty set we denote the thin spider and the thick spider by $\operatorname{thin}_{k}$ and thick $k$, respectively. Clearly, the complement of a thin spider is a thick spider, and vice versa. A fat spider is obtained from a spider by adding a true or false twin of a vertex $v \in S \cup C$. The following theorem characterizes $P_{4}$-tidy graphs.

Theorem 1 [8] Let $G$ be a $P_{4}$-tidy graph with at least two vertices. Then, exactly one of the following conditions holds:

\section{G is disconnected.}

2. $\bar{G}$ is disconnected.

3. $G$ is isomorphic to $P_{5}, \overline{P_{5}}, C_{5}$, a spider, or a fat spider.

Tree-cographs [20] are another generalization of cographs. They are defined recursively as follows: trees are tree-cographs; the disjoint union of tree-cographs is a tree-cograph; and the complement of a tree-cograph is also a tree-cograph.

The problem of characterizing interval graphs by forbidden induced subgraphs was solved by Boland and Lekkerkerker in [15].

Theorem 2 [15] A graph is an interval graph if and only if it contains no induced bipartite-claw, umbrella, $n$-net for any $n \geq 2$, $n$-tent for any $n \geq 3$, or $C_{n}$ for any $n \geq 4$.

Roberts was able to prove that an interval graph is unit if and only if it is claw-free [18]. Combining this result and the above theorem, the characterization below by forbidden induced subgraphs for unit interval graphs follows.

Corollary 3 A graph is a unit interval graph if and only if it contains no induced claw, 2-net, 2-tent, or $C_{n}$ for any $n \geq 4$. 
For other characterizations of unit interval graphs, the reader is referred to the works of Roberts [18] and Wegner [21].

Probe interval and probe unit interval graphs have been already characterized by forbidden induced subgraphs within the trees. In this work, we present generalizations of these theorems, which characterize in terms of forbidden induced subgraphs probe interval graphs and probe unit interval graphs within treecographs (see Sections 4 and 7 ).

Theorem 4 [19] Let $G$ be a tree. Then $G$ is a probe interval graph if and only if $G$ contains no induced $\Pi_{1}$ or $\Pi_{2}$.

Theorem 5 [3] Let $G$ be a tree. Then $G$ is a probe unit interval graph if and only if $G$ contains no induced bipartite-claw, $L$, or $H_{n}$ for any $n \geq 0$.

The length of an induced cycle is the number of its edges. The following lemma shows an infinite family of forbidden induced subgraphs for the class of probe interval graphs.

Lemma 6 [10] No odd cycle of length at least five is probe interval.

Notice that this result is also true if we replace probe interval by probe unit interval.

Let $\mathcal{K}$ be the class of graphs formed by the complete graphs. Let us study the class $\mathcal{P}(\mathcal{K})$, used throughout this article.

Lemma 7 A graph $G$ is probe $\mathcal{K}$ if and only if $G$ does not contain any induced $C_{4}$ or $\bar{P}_{3}$.

Proof: It is easy to see that neither $C_{4}$ nor $\bar{P}_{3}$ is a probe $\mathcal{K}$ graph. Conversely, if $G$ is a $\left\{\bar{P}_{3}, C_{4}\right\}$-free graph, then $\bar{G}$ is a complete graph plus a disjoint union of isolated vertices. So, $G$ can be partitioned into a stable set and a clique in such a way that every vertex of the stable set is adjacent to every vertex of the clique. Therefore, $G$ is a probe complete graph.

We will also refer to a probe complete graph as a split complete graph.

\section{Co-bipartite graphs and trees}

In this section we present a forbidden induced subgraph characterization for probe interval graphs within the class of those graphs whose complement is bipartite. Furthermore, we show that, restricted to the class of co-bipartite graphs, probe interval graphs, probe $\left\{3 K_{1}, C_{4}, C_{5}\right\}$-free graphs and probe unit interval graphs are the same classes. The class of probe $\left\{3 K_{1}, C_{4}, C_{5}\right\}$-free graphs will play a very important role in the following sections. The main results of this section are Theorem 10 which implies Corollary 11 (a result used to characterize probe interval graphs and probe unit interval graphs within the class of complement of trees) and Theorem 12 that characterizes $\left\{3 K_{1}, C_{4}, C_{5}\right\}$-free graphs within the class of complement of trees. Theorem 12 will play a central role in the characterization of probe unit interval graphs within the class of tree-cographs (cf. Section 7).

Given a graph $G$, a set $D \subseteq V(G)$ is called a dominating set if every vertex $v \in V(G)$ either belongs to $D$ or is adjacent to a vertex in $D$.

Lemma 8 [16] Let $G$ be a triangle-free graph. Then $G$ is $\left\{P_{6}, C_{6}\right\}$-free if and only if every induced connected subgraph of $G$ has a dominating complete bipartite subgraph isomorphic to $K_{n, m}$ with $n, m \geq$ 1 . 
The following lemma follows from Lemma 8

Lemma 9 Let $G=(V, E)$ be a connected bipartite $\left\{2 P_{3}, 3 K_{2}, P_{6}, C_{6}, Z\right\}$-free graph. Then, either $G$ has diameter at most 3 , or there exists a pendant vertex $v \in V$ such that $H=G-v$ has diameter at most 3.

Proof: Let $G$ be a connected bipartite $\left\{2 P_{3}, 3 K_{2}, P_{6}, C_{6}, Z\right\}$-free graph. By Lemma 8 , there exists a dominating complete bipartite graph $H=\left(V^{\prime}, E^{\prime}\right)$ such that $V^{\prime}$ can be partitioned into two stable sets $A$ and $B$. Since $G$ is bipartite, either $N_{H}(v) \cap A=\emptyset$ or $N_{H}(v) \cap B=\emptyset$ for every vertex $v \in V-(A \cup B)$. We will call $A^{\prime}$ the set of vertices of $V-V^{\prime}$ whose neighbors belong to $A$ and $B^{\prime}$ the set of vertices of $V-V^{\prime}$ whose neighbors belong to $B$. Assume that there exist two vertices $u, v \in V$ such that $d(u, v)=4$. Notice that either $u, v \in A^{\prime}$ or $u, v \in B^{\prime}$. Suppose without loss generality, that $u, v \in A^{\prime}$, let $u^{\prime} \in A$ and $v^{\prime} \in A$ be a neighbor of $u$ and $v$, respectively. Since $G$ is $Z$-free, $\bigcap_{b^{\prime} \in B^{\prime}} N\left(b^{\prime}\right) \neq \emptyset$; i.e., all vertices in $B^{\prime}$ have a common neighbor. Consequently, given a vertex $b^{\prime} \in B^{\prime}, d\left(b^{\prime}, z\right) \leq 3$ for all vertices $z \in V$. On the other hand, since $G$ is $3 K_{2}$-free, every vertex $w \in A^{\prime}$ is either adjacent to $u^{\prime}$ or adjacent to $v^{\prime}$. Since $G$ is $2 P_{3}$-free, either $u$ or $v$ is a pendant vertex. If $A^{\prime}=\{u, v\}, u$ or $v$ would satisfy the condition of the lemma. So, we can assume that $A^{\prime}-\{u, v\} \neq \emptyset$. Suppose, without loss of generality that $u$ is the pendant vertex. Since $G$ is $2 P_{3}$-free, if $u_{1} \in A^{\prime}-\{u\}$ is adjacent to $u^{\prime}$ and $v_{1} \in A^{\prime}-\{v\}$ is adjacent to $v^{\prime}$, then $u_{1}$ is adjacent to $v^{\prime}$ or $v_{1}$ is adjacent to $u^{\prime}$. Consequently, $u$ is a pendant vertex satisfying the conditions of the lemma.

Lemma9 9 implies the following characterization.

Theorem 10 Let $G$ be a co-bipartite graph. Then, the following statements are equivalent:

1. G is a probe interval graph;

2. $G$ is a probe unit interval graph;

3. $G$ is probe $\left\{3 K_{1}, C_{4}, C_{5}\right\}$-free;

4. $G$ is $\left\{\overline{2 P_{3}}, \overline{3 K_{2}}, \overline{P_{6}}, \overline{C_{6}}, \bar{Z}\right\}$-free.

Proof: It is easy to see that $\overline{2 P_{3}}, \overline{3 K_{2}}, \overline{P_{6}}, \overline{C_{6}}$ and $\bar{Z}$ are neither probe interval, nor probe unit interval, nor probe $\left\{3 K_{1}, C_{4}, C_{5}\right\}$-free.

Conversely, let $G=(V, E)$ be a $\left\{\overline{2 P_{3}}, \overline{3 K_{2}}, \overline{P_{6}}, \overline{C_{6}}, \bar{Z}\right\}$-free co-bipartite graph. Consider the complement graph of $G, \bar{G}$. By Theorem 2 , if $\bar{G}$ had diameter at most 3; i.e., $\bar{G}$ were $2 K_{2}$-free, then $G$ would be an interval graph. Therefore, we can assume that $\bar{G}$ has diameter 4 . By Lemma 9 , there exists a pendant vertex $v \in V$, whose neighbor we will call $v^{\prime}$, such that $H=\bar{G}[V-v]$ has diameter at most 3. Consequently, the completion $G^{*}(N \cup P, E \cup F)$, where $N=\left\{v, v^{\prime}\right\}, P=V-N$ and $F=\left\{v v^{\prime}\right\}$, is an interval graph. Finally, since $G^{*}$ is also co-bipartite and interval, $G$ is $\left\{C_{4}, C_{5}\right\}$-free, $3 K_{1}$-free (consequently, claw-free) and thus $\left\{3 K_{1}, C_{4}, C_{5}\right\}$-free and unit interval. Therefore, $G$ is probe $\left\{3 K_{1}, C_{4}, C_{5}\right\}$-free and probe unit interval.

As a consequence of Lemma 9 , we obtain the following corollary.

Corollary 11 Let $G$ be the complement of a tree. Then the following assertions are equivalent: 
1. $G$ is a probe $\left\{3 K_{1}, C_{4}, C_{5}\right\}$-free graph.

2. G is a probe unit interval graph.

3. G is a probe interval graph.

4. $G$ is $\left\{\overline{3 K_{2}}, \overline{2 P_{3}}, \overline{P_{6}}\right\}$-free.

5. $G$ is $\left\{\right.$ co-bipartite-claw, $\left.\bar{Q}, \overline{P_{6}}\right\}$-free.

(Here, item 4 is a minimal forbidden induced subgraph characterization, while item 5. is a minimal forbidden connected induced subgraph characterization.)

Proof: The equivalence between the first four statements follows from Theorem 10 .

Let us see the equivalence between 4 . and 5. Since $\bar{G}$ is $\left\{3 K_{2}, 2 P_{3}, P_{6}\right\}$-free, then $\bar{G}$ is $\{$ bipartite-claw, $\left.H, P_{6}\right\}$-free. Conversely, let $\bar{G}$ be a $P_{6}$-free tree. If $\bar{G}$ contains an induced $2 P_{3}$ or an induced $3 K_{2}$, then $\bar{G}$ contains either an induced $H$ or an induced bipartite-claw.

The following theorem gives a forbidden induced subgraph characterization of probe $\left\{3 K_{1}, C_{4}, C_{5}\right\}$ free graphs among trees. The class of probe $\left\{3 K_{1}, C_{4}, C_{5}\right\}$-free graphs will be useful in the following sections when dealing with the class of probe unit interval graphs. The graph E, mentioned in the following statement, is the graph obtained from the bipartite-claw by deleting a vertex of degree one.

Theorem 12 Let $G$ be a tree. Then the following assertions are equivalent:

1. G is a probe $\left\{3 K_{1}, C_{4}, C_{5}\right\}$-free graph.

2. G contains no induced $2 K_{2}+K_{1}$ or $P_{4}+K_{1}$.

3. G contains no induced $E$ or $P_{6}$.

(Here, item 2 is a minimal forbidden induced subgraph characterization, while item 3. is a minimal forbidden connected induced subgraph characterization.)

Proof: We first prove the equivalence of 1 . and 3 .. It is straightforward to verify that neither $E$ nor $P_{6}$ is a probe $\left\{3 K_{1}, C_{4}, C_{5}\right\}$-free graphs. Conversely, suppose that $G$ is $\left\{E, P_{6}\right\}$-free. Let $P=v_{1} v_{2} v_{3} \cdots v_{n}$ be a chordless path of maximum length of $G$. Since $G$ is a tree and $P$ is of maximum length, $v_{1}$ and $v_{n}$ are pendant vertices of $G$. Since $G$ contains no induced $P_{6}, n \leq 5$. Since $G$ is an $E$-free tree and $P$ is of maximum length, for each $i \in\{2, \ldots, n-1\}$, the neighbors of $v_{i}$ in $G$ different from $v_{i-1}$ and $v_{i+1}$ are pendant vertices of $G$. If $n \leq 3, G$ is probe complete and, in particular, it is a probe $\left\{3 K_{1}, C_{4}, C_{5}\right\}$-free graph. So, assume that $4 \leq n \leq 5$. If $n=5$, then $d_{G}\left(v_{3}\right)=2$ because $G$ contains no induced $E$. Let $N_{1}=N_{G}\left(v_{2}\right)-\left\{v_{3}\right\}$ and let $N_{2}=N_{G}\left(v_{n-1}\right)-\left\{v_{2}\right\}$ (so, if $n=5, N_{2}=N_{G}\left(v_{n-1}\right)$ ). Hence, we split $V(G)$ into $N=N_{1} \cup N_{2}$ which is clearly a stable set of $G$ and $P=V(G)-N$. The graph $G^{*}$ that arises from $G$ by adding all the edges joining two vertices of $N_{1}$ and all the edges joining two vertices of $N_{2}$ is $3 K_{1}$-free and chordal. Thus, $G^{*}$ is a $\left\{3 K_{1}, C_{4}, C_{5}\right\}$-free completion of $G$.

We now prove the equivalence of 2 . and 3 .. It can be easily seen that if $G$ is $\left\{2 K_{2}+K_{1}, P_{4}+K_{1}\right\}$-free, then $G$ does not contain any induced $E$ and $P_{6}$. Conversely, if $G$ is $P_{6}$-free and contains either an induced $2 K_{2}+K_{1}$ or an induced $P_{4}+K_{1}$ and $G$ is a tree and thus connected, then it contains an induced $E$. Consequently, if $G$ is a $\left\{E, P_{6}\right\}$-free tree, then $G$ contains no induced $2 K_{2}+K_{1}$ or $P_{4}+K_{1}$. 


\section{Probe interval graphs}

Given two graphs $G$ and $H$, the join of $G$ and $H$, denoted $G \vee H$, is the graph obtained from the disjoint union of $G$ and $H$ by adding the edges $\{u v: u \in V(G), v \in V(H)\}$.

Lemma 13 Let $G_{1}$ and $G_{2}$ be two graphs. Then $G_{1} \vee G_{2}$ is an interval graph if and only if one of $G_{1}$ and $G_{2}$ is interval and the other one is a complete graph.

Proof: Since interval graphs are a hereditary class, if $G_{1} \vee G_{2}$ is an interval graph then $G_{1}$ and $G_{2}$ are interval graphs. Suppose that none of $G_{1}$ and $G_{2}$ is a complete graph. Then, there exist two nonadjacent vertices $v_{1}^{i}, v_{2}^{i} \in V\left(G_{i}\right)$ for $i=1,2$. Consequently, $\left\{v_{1}^{1}, v_{2}^{1}, v_{1}^{2}, v_{2}^{2}\right\}$ induces $C_{4}$ in $G_{1} \vee G_{2}$ and thus $G_{1} \vee G_{2}$ is not interval. Conversely, suppose that $G_{1}$ or $G_{2}$ is interval and the other one a complete graph, say $G_{1}$ is interval and $G_{2}$ is a complete graph. So, we can construct an interval model for $G_{1} \vee G_{2}$ from the interval model $\mathcal{S}$ of $G_{1}$ by adding as many intervals as the number of vertices of $G_{2}$, covering the whole interval model $\mathcal{S}$.

Lemma 14 Let $G_{1}$ and $G_{2}$ be two graphs. Then $G_{1} \vee G_{2}$ is a probe interval graph if and only if one of the following assertions holds:

1. One of $G_{1}$ and $G_{2}$ is a complete graph and the other one is probe interval.

2. One of $G_{1}$ and $G_{2}$ is probe complete and the other one is interval.

Proof: Let $G_{1}$ and $G_{2}$ be two graphs and let $H=G_{1} \vee G_{2}$ be probe interval. Therefore, there exists a probe interval completion $H^{*}=(N \cup P, E \cup F)$ of $H$ such that $H^{*}$ is an interval graph. Since $H=G_{1} \vee G_{2}$, either $N \subseteq V\left(G_{1}\right)$ or $N \subseteq V\left(G_{2}\right)$. Assume, without loss of generality, that $N \subseteq V\left(G_{1}\right)$; i.e., $H^{*}=G_{1}^{*} \vee G_{2}$ with $G_{1}^{*}=\left(V\left(G_{1}\right), E\left(G_{1}\right) \cup F\right)$. By Lemma 13, since $H^{*}$ is an interval graph, one of $G_{1}^{*}$ and $G_{2}$ is a complete graph and the other one is interval. So, either $G_{1}$ is probe complete and $G_{2}$ is an interval graph or $G_{1}$ is a probe interval and $G_{2}$ is a complete graph.

Notice the following immediate class inclusion:

complete $\subseteq$ probe complete $\subseteq$ interval $\subseteq$ probe interval.

The following theorem characterizes all minimal non-(probe interval) graphs whose complement is disconnected.

Theorem 15 The only minimally non-(probe interval) graphs whose complement is disconnected are bipartite-claw $\vee 2 K_{1}$, umbrella $\vee 2 K_{1}, n$-net $\vee 2 K_{1}$ where $n \geq 2, n$-tent $\vee 2 K_{1}$ for any $n \geq 3, \overline{3 K_{2}}$, and $\overline{2 P_{3}}$.

Proof: Let $G$ be a minimally non-(probe interval) graph whose complement is disconnected. Since $\bar{G}$ is disconnected, there exist two graphs $G_{1}$ and $G_{2}$ such that $G$ is the join between them; i.e., $G=G_{1} \vee G_{2}$. By minimality, $G_{1}$ and $G_{2}$ are probe interval. Since $G=G_{1} \vee G_{2}$ is non-(probe interval), by Lemma13. none of $G_{1}$ and $G_{2}$ is a complete graph.

Suppose that one of $G_{1}$ and $G_{2}$ is probe complete, say $G_{2}$. Then, $G_{1}$ is not interval because otherwise $G_{1} \vee G_{2}$ would be probe interval. Since, for each $v_{1} \in V\left(G_{1}\right),\left(G_{1}-v_{1}\right) \vee G_{2}$ is probe interval and $G_{2}$ is 


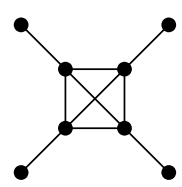

thin $_{4}$

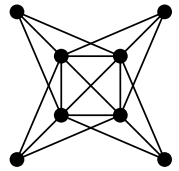

thick $_{4}$

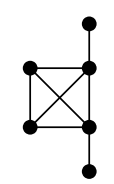

$\operatorname{thin}_{2}\left(K_{2}\right)$

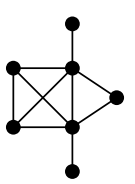

$\operatorname{thin}_{2}\left(K_{2} \cup K_{1}\right)$

Fig. 2: Some spiders.

not a complete graph, $G_{1}-v_{1}$ is an interval graph. Thus, $G_{1}$ is a minimally non interval graph. Since, for each $v_{2} \in V\left(G_{2}\right), G_{1} \vee\left(G_{2}-v_{2}\right)$ is probe interval and $G_{1}$ is not interval, $G_{2}-v_{2}$ is a complete graph. Since $G_{2}$ is not a complete graph, $G_{2}=2 K_{1}$. Since $G_{1}$ is probe interval, $G_{1}$ is not a cycle of length at least 5 (see Lemma 6). We conclude that $G$ equals bipartite-claw $\vee 2 K_{1}$, umbrella $\vee 2 K_{1}, n$-net $\vee 2 K_{1}$ for some $n \geq 2, n$-tent $\vee 2 K_{1}$ for some $n \geq 3$, or $C_{4} \vee 2 K_{1}=\overline{3 K_{2}}$.

We can now assume that $G_{1}$ and $G_{2}$ are non-(probe complete). Since $\left(G_{1}-v_{1}\right) \vee G_{2}$ is probe interval, $G_{1}-v_{1}$ is probe complete, for each $v_{1} \in V\left(G_{1}\right)$. So $G_{1}$ is a minimally non-(probe complete), i.e., $\overline{P_{3}}$ or $C_{4}$ (see Lemma 7). Symmetrically, $G_{2}$ is $\overline{P_{3}}$ or $C_{4}$. If $G_{1}=C_{4}$ or $G_{2}=C_{4}$, then $G$ contains a proper induced $C_{4} \vee 2 K_{1}$, a contradiction. Therefore $G=\overline{2 P_{3}}$.

The following theorem characterizes those probe interval graphs among tree-cographs.

Theorem 16 Let $G$ be a tree-cograph. Then, $G$ is a probe interval graph if and only if $G$ contains no induced $\Pi_{1}, \Pi_{2}$, bipartite-claw $\vee 2 K_{1}, \overline{3 K_{2}}, \overline{2 P_{3}}$, or $\overline{P_{6}}$.

Proof: It suffices to prove that if $G$ is a tree-cograph non-(probe interval) graph, then $G$ contains an induced $\Pi_{1}, \Pi_{2}$, bipartite-claw $\vee 2 K_{1}, \overline{3 K_{2}}, \overline{2 P_{3}}$, or $\overline{P_{6}}$.

So, assume that $G$ is a tree-cograph that is not probe interval. Therefore, $G$ contains an induced subgraph $H$ that is a minimally non-(probe interval) graph. Since $G$ is a tree-cograph, $H$ is also a treecograph. Consequently, $H$ is disconnected, or the complement of $H$ is disconnected, or $H$ is a tree, or $H$ is the complement of a tree. By minimality of $H, H$ is not disconnected because the disjoint union of probe interval graphs is also a probe interval graph. If the complement of $H$ is disconnected, then (by Theorem 15 $H$ equals bipartite-claw $\vee 2 K_{1}, \overline{3 K_{2}}$ or $\overline{2 P_{3}}$ (notice that umbrella $\vee 2 K_{1}, n$-net $\vee 2 K_{1}$ for any $n \geq 2$, and $n$-tent $\vee 2 K_{1}$ for any $n \geq 3$ are not tree-cographs). If $H$ is a tree, Theorem 4 implies that $H$ equals $\Pi_{1}$ or $\Pi_{2}$. Finally, consider the case when $H$ is the complement of a tree. By Theorem $11 . H$ equals $\overline{3 K_{2}}, \overline{2 P_{3}}$, or $\overline{P_{6}}$.

In order to characterize those probe interval graphs within $P_{4}$-tidy graphs, we need the following lemma that characterizes those spiders that are probe interval.

Lemma 17 Let $H$ be a spider with spider partition $(C, S, R)$. Then, $H$ is probe interval if and only if one of the following conditions holds:

1. $|C|=3$ and $H[R]$ is interval.

2. $|C|=2$ and $H[R]$ is probe interval.

Moreover, if $H$ is probe interval, then a fat spider $H^{\prime}$ that arises from $H$ is also probe interval except when $|C|=2, H^{\prime}$ arises by making a false twin of a vertex of $C$, and $H[R]$ is not interval. 
Proof: Let $H=(V, E)$ be a thick (thin) spider with partition $(C, S, R)$ that is probe interval with a completion $H^{*}=(V, E \cup F)$ and probe interval partition $(N, P)$. Suppose that $|C| \geq 4$ and let $c_{1}, c_{2}, c_{3}, c_{4}$ be different vertices in $C$. Notice that if a tent (net) is an induced subgraph of $H$, then exactly a vertex of degree four (three) belongs to $N$. Let $s_{1}, s_{2}, s_{3}, s_{4}$ be different vertices of $S$ such that $s_{i}$ adjacent to any vertex in $C$ but $c_{i}$ ( $s_{i}$ is only adjacent to $c_{i}$ ) for all $1 \leq i \leq|C|$. So, $\left\{c_{1}, c_{2}, c_{3}, s_{1}, s_{2}, s_{3}\right\}$ induces a tent (net) and thus one of $c_{1}, c_{2}, c_{3}$ belongs to $N$, say $c_{1}$. Analogously, $\left\{c_{2}, c_{3}, c_{4}, s_{2}, s_{3}, s_{4}\right\}$ also induces a tent (net) and one of $c_{2}, c_{3}, c_{4}$ belongs to $N$, but all all graphs of the lemma are adjacent to $c_{1}$, a contradiction. Consequently, $2 \leq|C| \leq 3$. Assume that $|C|=3$. Since $H$ is probe interval, $H[C+R]=H[C] \vee H[R]$ is also probe interval. On the one hand, since $H[C]$ is a complete graph, by Lemma $14 H[R]$ is probe interval. On the other hand, since $\left\{s_{1}, s_{2}, s_{3}, c_{1}, c_{2}, c_{3}\right\}$ induces a tent (net), one of the vertices in $C$ is nonprobe and thus any vertex in $R$ is probe. So, $H[R]$ is interval. Now, assume that $|C|=2$. Since $C$ is a complete graph and $H$ is probe interval, $H[C] \vee H[R]$ is probe interval. Thus, by Lemma $14, H[R]$ is probe interval. Conversely, it is straightforward to construct a probe interval model of a thick (thin) spider that satisfies condition 1 . or 2 ..

Let $H^{\prime}$ be a fat spider that arises from $H$. If $H^{\prime}$ arises by making a twin of a vertex $s \in S$, then $H^{\prime}$ is also probe interval. Indeed, if $H^{*}=(V(H), E(H) \cup F)$ is a probe interval completion of $H$ with a probe interval partition $(N, P)$ chosen (by symmetry) in such a way that $s \in N$ if $s^{\prime}$ is a false twin and $s \in P$ if $s^{\prime}$ is a true twin, then $(N, P)$ can be extended to a probe interval partition $\left(N^{\prime} P^{\prime}\right)$ of $H^{\prime}$ by taking also the twin $s^{\prime}$ of $s$ as a nonprobe vertex $\left(N^{\prime}=N \cup\{s\}\right)$ if it is a false twin and as a probe vertex $\left(P^{\prime}=P \cup\{s\}\right)$ if it is a true twin. Therefore, $H^{\prime *}=\left(N^{\prime} \cup P, E\left(H^{\prime}\right) \cup F^{\prime}\right)$, with $F^{\prime}=F \cup\left\{s s^{\prime}\right\} \cup\left\{v s^{\prime}: v s \in F\right\}$ if $s^{\prime}$ is a false twin and $F^{\prime}=F$ if $s^{\prime}$ is a true twin, is interval because the graph obtained by adding true twins to an interval graph is also interval. Suppose now that $H^{\prime}$ arises from a vertex $c \in C$ by making a true twin. Then $H^{\prime}$ is probe interval. In fact, any partition $(N, P)$ of $H$ where $c \in P$ can be extended to a partition of $H^{\prime}$ where the new vertex is also a probe vertex. Finally, consider the case where $H$ arises by making a false twin of a vertex $c \in C$. If $H[R]$ were not interval and thus $|C|=2$, then $H$ would contain $H[R] \vee 2 K_{1}$, where $H[R]$ is a forbidden induced subgraph for the class of interval graphs. Therefore, $H^{\prime}$ would not be probe interval. Notice that if $H[R]$ is interval, then clearly $H^{\prime}$ is an interval, simply look for a partition where $c$ and the false twin of it are both nonprobe, and the vertices of $R$ are all probe.

Let $\mathcal{H}$ be the set formed by all the minimally non interval graphs except for the induced cycles with at least five vertices. Graphs belonging to $\mathcal{H}$ are probe interval. In addition, it can be proved that every probe interval partition of a graph belonging to $\mathcal{H}$ contains at least two nonadjacent probe interval vertices. Therefore, graphs arising from a graph belonging to $\mathcal{H}$ by adding two nonadjacent universal vertices is a minimally non-(probe interval).

Lemma 18 The minimally non-(probe interval) graphs that are spiders or fat spiders are the graphs: thick $_{3}(H)$, $\operatorname{thin}_{3}(H)$, for $H \in \mathcal{H}$, thin ${ }_{4}$ and thick 4 .

Proof: It is straightforward to check that all the graphs of the lemma are minimally non-(probe interval). Let $G$ be a thick (thin) spider with partition $(C, S, R)$ that is minimally non-(probe interval). If $|C|$ were of size at least 4 , then $G$ would contain thick (thin $_{4}$ ) as induced subgraph and by minimality $G=$ thick $_{4}$ $\left(G=\operatorname{thin}_{4}\right)$. Now, we may assume that $|C| \leq 3$. Indeed, by Lemma 17 , since $G$ is non-(probe interval), it suffices to consider $|C|=3$. By minimality, $G[R]$ is probe interval and by Lemma 17 it cannot be an interval graph. By Theorem 2, the only minimal not interval graphs that are probe interval are 
those graphs belonging to $\mathcal{H}$. Therefore, $G$ contains $\operatorname{thick}_{3}(H)\left(\operatorname{thin}_{3}(H)\right)$ for some $H \in \mathcal{H}$ as induced subgraph. Furthermore, by minimality, $G$ is exactly that graph.

Let $G$ be a fat spider that is a minimally non-(probe interval). By Lemma 17, $G$ arises from a spider with $|C|=2$ and $H[R]$ is not an interval graph by making a false twin of a vertex in $C$. Assume that $C=\left\{c_{1}, c_{2}\right\}, S=\left\{s_{1}, s_{2}\right\}$ and $v$ is a false twin of $c_{1}$. By minimality $G-v$ is probe interval. By Lemma 17 and minimality, $H[R]$ is probe interval but it is not an interval graph. Consequently, $H[R]$ contains an induced minimally non interval graph $W$. By minimality, $W \in \mathcal{H}$. Since $v$ is not adjacent to $c_{1}$ and they are complete to $W, G$ contains an induced $U=W \vee 2 K_{2}$ with $W \in \mathcal{H}$. By Lemma 14 it follows that $U$ is minimally non-(probe interval). By minimality, $G=U$, this leads to a contradiction because $U$ is not a spider.

Theorem 19 Let $G$ be a $P_{4}$-tidy graph. Then $G$ is a probe interval graph if and only if $G$ contains no

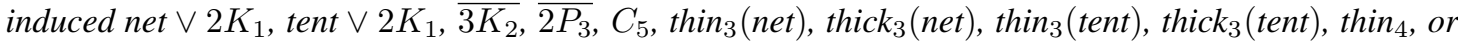
thick $_{4}$.

Proof: Let $G$ be a minimally non-(probe interval) graph that is a $P_{4}$-tidy graph. By minimality, $G$ is connected. If $\bar{G}$ were disconnected then, by Theorem 15 . $G$ would be isomorphic to either net $\vee 2 K_{1}$, or tent $\vee 2 K_{1}$, or $\overline{3 K_{2}}$, or $\overline{2 P_{3}}$. So, we may assume that $\bar{G}$ is connected. By Theorem $1 . G$ is $C_{5}, P_{5}, \overline{P_{5}}$, a spider or a fat spider. Notice that $P_{5}$ and $\overline{P_{5}}$ are probe interval graphs. So, $G$ is isomorphic to $C_{5}$ or, by Corollary 18, $G$ is isomorphic to either $\operatorname{thin}_{3}(H)$, or thick $3(H)$, for $H \in\{$ net, tent $\}$, or $\operatorname{thin}_{4}$, or thick 4 .

\section{Graphs classes with a companion}

Let $\mathcal{G}$ be a hereditary class. We say that a class $\mathcal{H}$ is the companion of $\mathcal{G}$ if and only if, given any two graphs $G_{1}$ and $G_{2}$, the following holds: $G_{1} \vee G_{2} \in \mathcal{G}$ if and only if one of $G_{1}$ and $G_{2}$ is a complete graph and the other one belongs to $\mathcal{H}$.

For example, by Lemma 13 , the class of interval graphs is its own companion. Using the Robert's characterizations for unit interval graphs [18] it follows that the companion of the class of unit interval graphs is the class of $\left\{3 K_{1}, C_{4}, C_{5}\right\}$-free graphs. Notice also that the companion of the class $\left\{3 K_{1}, C_{4}, C_{5}\right\}$-free is itself.

The main result of this section is Theorem 22 This theorem allows to compute all the minimally non-(probe $\mathcal{G}$ ) graphs whose complement graph is disconnected, being $\mathcal{G}$ a hereditary graph class with a companion.

Lemma 20 Let $\mathcal{G}$ be a hereditary graph class and $\mathcal{H}$ be its companion. Then, the following assertions hold:

1. $\mathcal{K} \subseteq \mathcal{H} \subseteq \mathcal{G}$.

2. $\mathcal{H}$ is a hereditary class.

3. $\mathcal{H}$ is its own companion.

4. $C_{4} \notin \mathcal{G}$.

5. If $\mathcal{H} \neq \mathcal{K}$, then $C_{4}$ is a minimally non- $\mathcal{G}$ graph and a probe $\mathcal{H}$ graph. 
Proof: Let $H \in \mathcal{H}$. Since $\mathcal{H}$ is the companion of $\mathcal{G}, K_{n} \vee H \in \mathcal{G}$ for every positive integer $n$. Since $G$ is hereditary, $\mathcal{K} \subseteq \mathcal{G}$ and $\mathcal{H} \subseteq \mathcal{G}$. Since $\mathcal{K} \subseteq \mathcal{G}, K_{n} \vee K_{n} \in \mathcal{G}$ for every positive integer $n$, and therefore $\mathcal{K} \subseteq \mathcal{H}$. We conclude that $\mathcal{K} \subseteq \mathcal{H} \subseteq \mathcal{G}$. On the other hand, since $\mathcal{G}$ is an hereditary class $H^{\prime} \vee K_{1} \in \mathcal{G}$ for any $H^{\prime}$ subgraph of $H$. Therefore, $H^{\prime} \in \mathcal{K} \subseteq \mathcal{H}$ or $H^{\prime} \in \mathcal{H}$. Consequently, $\mathcal{H}$ is a hereditary class. 3 . is immediate from 1 . and the definition of hereditary class with a companion. $C_{4}$ does not belong to $\mathcal{G}$ because $C_{4}=2 K_{1} \vee 2 K_{1}$ and $2 K_{1} \notin \mathcal{K}$.

Assume now that $\mathcal{H} \neq \mathcal{K}$. Since $\mathcal{H}$ is hereditary, $2 K_{1} \in \mathcal{H}$ and consequently $P_{3}=K_{1} \vee 2 K_{1} \in \mathcal{G}$. Moreover, since $2 K_{1} \in \mathcal{H}, K_{1} \vee$ diamond $=K_{3} \vee 2 K_{1} \in \mathcal{G}$, which implies that diamond $\in \mathcal{H}$. Therefore, $C_{4}$ is a minimally non- $\mathcal{G}$ graph but it is a probe $\mathcal{H}$ graph.

The following lemma shows the behavior of the join operator with respect to a hereditary class with a companion.

Lemma 21 Let $\mathcal{G}$ be a hereditary graph class with companion $\mathcal{H}$ and let $G_{1}$ and $G_{2}$ be two nonempty graphs. Then, $G_{1} \vee G_{2}$ is a probe $\mathcal{G}$ graph if and only if at least one of the following conditions holds:

1. One of $G_{1}$ and $G_{2}$ is a complete graph and the other one is a probe $\mathcal{H}$-graph.

2. One of $G_{1}$ and $G_{2}$ is probe complete and the other one is an $\mathcal{H}$-graph.

Proof: The "if" part is straightforward. So, we are going to prove the "only if" part. Let $G=G_{1} \vee G_{2}$ be a probe $\mathcal{G}$ graph, with $\mathcal{G}$ a hereditary class with a companion $\mathcal{H}$. Therefore, there exists a completion $G^{*}=(V(G), E(G) \cup F)$ with a probe interval partition $(N, P)$ such that $G^{*} \in \mathcal{G}$. Since $N$ is an independent set, $N \subseteq V\left(G_{1}\right)$ or $N \subseteq V\left(G_{2}\right)$. Assume, without loss of generality, that $N \subseteq V\left(G_{1}\right)$ and we call $G_{1}^{*}$ to the graph whose vertex set is $V\left(G_{1}\right)$ and whose edge set is $E\left(G_{1}\right) \cup F$. Consequently, since $\mathcal{H}$ is the companion of $\mathcal{G}$, either $G_{1}^{*}$ is a complete graph $\left(G_{1}\right.$ is probe complete) or $G_{1}^{*} \in \mathcal{H}$ ( $G_{1}$ is probe $\mathcal{H})$ and $G_{2} \in \mathcal{H}$ or $G_{2}$ is a complete graph, respectively.

The following theorem gives a tool to calculate the minimally non-probe $\mathcal{G}$ graphs for a hereditary class $\mathcal{G}$ with a companion $\mathcal{H} \neq \mathcal{K}$.

Theorem 22 Let $\mathcal{G}$ be a hereditary graph class and $\mathcal{H}$ be its companion. If $\mathcal{H} \neq \mathcal{K}$ then the minimally non-(probe $\mathcal{G}$ ) graphs with disconnected complements are:

1. the graphs $W \vee K_{1}$ for each $W \in \mathcal{P}(G)$ that is minimally non-(probe $\left.\mathcal{H}\right)$;

2. the graphs $W \vee 2 K_{1}$ for each $W \in \mathcal{P}(G)$ that is minimally non- $\mathcal{X}$, where $\mathcal{X}=\mathcal{P}(\mathcal{K}) \cup \mathcal{H}$;

3. the graphs $W_{1} \vee W_{2}$ for each $W_{1}, W_{2} \in \mathcal{P}(\mathcal{K})$ that are minimally non- $\mathcal{H}$;

4. the graph $\overline{2 P_{3}}$.

Proof: Let $G$ be a minimally non-(probe $\mathcal{G}$ ) graph with disconnected complement. Then, $G=G_{1} \vee G_{2}$ where $G_{1}$ and $G_{2}$ are nonempty graphs.

Suppose that $G_{2}$ is a complete graph. Since $G_{1} \vee G_{2}$ is not a probe $\mathcal{G}$ graph, $G_{1}$ is not a probe $\mathcal{H}$ graph, see Lemma 21. By minimality and Lemma 21, $G_{2}$ is isomorphic to $K_{1}$ and thus $G_{1} \vee K_{1}$ is non-(probe $\mathcal{G})$. Since $G_{1}$ is not a probe $\mathcal{H}$ graph, in particular, $G_{1}$ is not a complete graph. Since $\left(G_{1}-v_{1}\right) \vee K_{1}$ is probe $\mathcal{G}, G_{1}-v_{1}$ is a probe $\mathcal{H}$ graph for each $v_{1} \in V\left(G_{1}\right)$. So, $G$ is isomorphic to $W \vee K_{1}$ where $W$ is 
minimally non-(probe $\mathcal{H}$ ) and a probe $\mathcal{G}$ graph. In what follows, we can assume that $G_{1}$ and $G_{2}$ are not complete graphs.

Suppose that $G_{2}$ contains an induced $C_{4}$. Since $\left(G_{1}-v_{1}\right) \vee C_{4}$ is probe $\mathcal{G}$ and $C_{4}$ is neither an $\mathcal{H}$ graph nor probe complete, then $G_{1}-v_{1}$ is a complete graph for each $v_{1} \in V\left(G_{1}\right)$. So, by Lemma 21, since $G_{1}$ is not a complete graph, $G_{1}$ is isomorphic to $2 K_{1}$. Since $C_{4} \vee 2 K_{1}$ is not a probe $\mathcal{G}$ graph, by minimality $G=C_{4} \vee 2 K_{1}$. Notice that $C_{4}$ is minimally non- $\mathcal{X}$ and probe $\mathcal{G}$. In what follows, we can assume that $G_{1}$ and $G_{2}$ contain no induced $C_{4}$.

Suppose that $G_{2}$ is probe complete and an $\mathcal{H}$ graph. Since $G_{1} \vee G_{2}$ is not a probe $\mathcal{G}$ graph, $G_{1}$ is not a $\mathcal{X}$ graph. Since $\left(G_{1}-v_{1}\right) \vee G_{2}$ is probe a $\mathcal{G}$ graph, $G_{1}-v_{1}$ is a $\mathcal{X}$ graph. So, $G_{1}$ is minimally non- $\mathcal{X}$ graph. Since $G_{1} \vee\left(G_{2}-v_{2}\right)$ is a probe $\mathcal{G}$ graph, $G_{2}-v_{2}$ is a complete graph for each $v_{2} \in V\left(G_{2}\right)$. Since $G_{2}$ is not a complete graph, $G_{2}=2 K_{1}$. So, $G=W \vee 2 K_{1}$ where $W$ is a minimally non- $\mathcal{X}$ graph that is probe $\mathcal{G}$.

Suppose that $G_{2}$ is probe complete but it is not an $\mathcal{H}$ graph. Since $G_{1} \vee G_{2}$ is not a probe $\mathcal{G}$ graph, $G_{1}$ is not an $\mathcal{H}$ graph. Since $\left(G_{1}-v_{1}\right) \vee G_{2}$ is a probe $\mathcal{G}$ graph, $G_{1}-v_{1}$ is an $\mathcal{H}$ graph. So, $G_{1}$ is minimally non- $\mathcal{H}$. Suppose, by way of contradiction, that $G_{1}$ is non-(probe complete). Since $G_{1} \vee\left(G_{2}-v_{2}\right)$ is a probe $\mathcal{G}$ graph, $G_{2}-v_{2}$ is a complete graph for each $v_{2} \in V\left(G_{2}\right)$. Since $G_{2}$ is not a complete graph, $G_{2}$ is isomorphic to $2 K_{1}$. Since $G_{2}$ is not an $\mathcal{H}$ graph, then $\mathcal{H} \subseteq \mathcal{K}$, a contradiction. The contradiction arose by assuming that $G_{1}$ is non-(probe complete). Therefore, $G_{1}$ is probe complete. Since $G_{1}$ is not an $\mathcal{H}$ graph, by symmetry, $G_{2}$ is a minimally non- $\mathcal{H}$ graph. We conclude that $G=W_{1} \vee W_{2}$ where $W_{1}$ and $W_{2}$ are minimally non- $\mathcal{H}$ graphs and probe complete.

Finally, we can assume that $G_{1}$ and $G_{2}$ are non-(probe complete). Since $G_{1}$ and $G_{2}$ contain no induced $C_{4}$, by Lemma 7, $G_{1}$ and $G_{2}$ contain an induced $\overline{P_{3}}$ each. By minimality, $G$ is isomorphic to $\overline{2 P_{3}}$.

Notice that Theorem 15 follows easily from the above theorem. Indeed, by Lemma 13 , the class $\mathcal{I}$ of interval graphs is the companion of itself. Since $\mathcal{P}(\mathcal{K}) \subseteq \mathcal{I}, \mathcal{P}(\mathcal{K}) \cup \mathcal{I}=\mathcal{I}$ and none of the minimally non- $\mathcal{I}$ graphs is probe complete, the only minimally non- $\mathcal{I}$ graphs that are non-(probe $\mathcal{I})$ graphs are the cycles $C_{n}$ for each $n \geq 5$.

Remark 1 If $\mathcal{H}=\mathcal{K}$, the graphs belonging to $\mathcal{G}$ are $P_{3}$-free (i.e., are the disjoint union of complete graphs) and the minimally non-(probe $\mathcal{G})$ graphs with disconnected complement are $C_{4}$ and $\overline{P_{3}}$, if $\overline{P_{3}} \notin \mathcal{G}$; or $C_{4}$ and paw, otherwise.

\section{Partial characterization of probe $\left\{3 K_{1}, C_{4}, C_{5}\right\}$-free graphs}

The main results of this section are Theorem 25 that characterizes probe $\left\{3 K_{1}, C_{4}, C_{5}\right\}$-free graphs within the class of tree-cographs and Theorem 28 that characterizes probe $\left\{3 K_{1}, C_{4}, C_{5}\right\}$-free graphs within the class of $P_{4}$-tidy graphs. They both will be used in the next section with Theorem 22 to characterize probe unit interval graphs among tree-cographs and $P_{4}$-tidy graphs.

Threshold graphs, introduced by Chvátal and Hammer in 1975 [7], can be defined as $\left\{2 K_{2}, P_{4}, C_{4}\right\}$ free graphs. Threshold graphs are a subclass of split graphs.

In what follows, $\mathcal{T}$ denotes the class of $\left\{3 K_{1}, C_{4}, C_{5}\right\}$-free graphs and $\mathcal{L}$ denotes the class $\mathcal{P}(\mathcal{K}) \cup \mathcal{T}$.

Lemma 23 The minimally non- $\mathcal{P}(\mathcal{T})$ disconnected graphs are $2 K_{2}+K_{1}, P_{4}+K_{1}$, and $C_{4}+K_{1}$.

Proof: It is straightforward to check that $2 K_{2}+K_{1}, P_{4}+K_{1}$, and $C_{4}+K_{1}$ are minimally non- $\mathcal{P}(\mathcal{T})$ graphs. 
Conversely, let $H$ be a disconnected minimally non- $\mathcal{P}(\mathcal{T})$ graph. Suppose, by way of contradiction, that $H$ does not contain $2 K_{2}+K_{1}, P_{4}+K_{1}$, and $C_{4}+K_{1}$ as induced subgraph. Consequently, $H$ is either a threshold graph or the union of two threshold graphs $H_{1}, H_{2}$ with no induced $K_{2}+K_{1}$ (i.e., split complete graphs).

In the first case, let $N$ be the stable set in the split partition of $H$. The graph $H^{*}$ that arises from $H$ by adding all the edges $u v$ with $u, v \in N$ is co-bipartite. So, $H^{*}$ is a completion of $H$ with partition $(N, P)(P=V(H)-N)$ that is $\left\{3 K_{1}, C_{5}\right\}$-free. Next, we will prove that $H^{*}$ is also $C_{4}$-free. Let $A=$ $\{u, v, x, y\}$ be a set of vertices of $H^{*}$ such that $H^{*}[A]$ is isomorphic to $C_{4}$. Notice that, by construction, we can assume that $u, v \in N, x, y \in P, u$ is adjacent to $x$ and $v$ is adjacent to $y$. But then $A$ induces a $P_{4}$ in $H$, a contradiction.

In the second case, let $N$ be the union of the stable sets in the split partitions of $H_{1}$ and $H_{2}$. Let $H^{*}$ be the graph that arises from $H$ by adding all the edges $u v$ with $u, v \in N$. Then $H^{*}$ can be obtained from $P_{4}$ by adding true twin vertices. It is easy to see then that it is a $\mathcal{T}$-graph.

As a consequence of Theorem 22 we can calculate all minimally non- $\mathcal{P}(\mathcal{T})$ graphs whose complement is disconnected.

Lemma 24 The minimally non- $\mathcal{P}(\mathcal{T})$ graphs with disconnected complement are $\left(K_{2}+2 K_{1}\right) \vee 2 K_{1}$, $\left(P_{3}+K_{1}\right) \vee 2 K_{1}, \overline{3 K_{2}}, K_{3,3}$, and $\overline{2 P_{3}}$.

Proof: Recall that the class $\mathcal{T}$ is its own companion. Let $W$ be a minimally non- $\mathcal{L}$ graph. We claim that $W$ isomorphic to either $K_{2}+2 K_{1}$, or $P_{3}+K_{1}$, or $C_{4}$, or $C_{5}$. Indeed, since $W$ is not a $\mathcal{T}$ graph, $W$ contains an induced $3 K_{1}, C_{4}$, or $C_{5}$. If $W$ contained an induced $C_{4}$ or $C_{5}$, then, by minimality, $W$ would be either isomorphic to $C_{4}$, or isomorphic to $C_{5}$. So, we may assume that $W$ contains an induced $3 K_{1}$ and no induced $C_{4}$ or $C_{5}$. Let $S$ be a set that induces a $3 K_{1}$ in $W$. Since $W$ is non-(probe complete) and $W$ contains no induced $C_{4}, W$ contains an induced $\overline{P_{3}}$ (see Lemma 7 ). Let $X$ be a set that induces a $\overline{P_{3}}$ in $W$ and $e=u v$ be the only edge joining two vertices of $X$ in $W$. If $e$ has one endpoint either in $S$ or adjacent to a vertex in $S$ (say $u$ ), then $W$ contains an induced $K_{2}+2 K_{1}$ or $P_{3}+K_{1}$, or $S \cup\{v\}$ induces a claw. If $W$ contains an induced $K_{2}+2 K_{1}$ or $P_{3}+K_{1}$, then, by minimality, $W$ is either isomorphic to $K_{2}+2 K_{1}$ or isomorphic to $P_{3}+K_{1}$. Suppose, by way of contradiction, that $W$ contains neither an induced $K_{2}+2 K_{1}$ nor an induced $P_{3}+K_{1}$. Then, $S \cup\{v\}$ induces a claw. Let $w$ be such that $X=\{u, v, w\}$. Since $W$ contains no induced $P_{3}+K_{1}, w$ is adjacent to both vertices of $S-\{u\}$ and consequently $W$ contains an induced $C_{4}$, a contradiction. Notice that $K_{2}+2 K_{1}, P_{3}+K_{1}, C_{4}$ are probe $\mathcal{T}$ graphs, but $C_{5}$ is not a probe $\mathcal{T}$ graph. Finally, the only minimally non- $\mathcal{T}$ graph that is probe complete is $3 K_{1}$. The results follows now from Theorem 22 .

As a consequence of Lemmas 23 and 24, we will present characterizations for probe $\mathcal{T}$ graphs within the classes of tree-cographs and $P_{4}$-tidy graphs.

Theorem 25 Let $G$ be a tree-cograph. Then, $G$ is a probe $\mathcal{T}$ graph if and only if $G$ contains no induced $2 K_{2}+K_{1}, P_{4}+K_{1}, C_{4}+K_{1},\left(K_{2}+2 K_{1}\right) \vee 2 K_{1},\left(P_{3}+K_{1}\right) \vee 2 K_{1}, \overline{3 K_{2}}, K_{3,3}, \overline{2 P_{3}}$, or $\overline{P_{6}}$.

Proof: Let $H$ be a minimally non- $\mathcal{P}(\mathcal{T})$ graph that is a tree-cograph. If $H$ is disconnected, then $H$ is $2 K_{2}+K_{1}, P_{4}+K_{1}$, or $C_{4}+K_{1}$. If $\bar{H}$ is disconnected, then $H$ is $\left(K_{2}+2 K_{1}\right) \vee 2 K_{1},\left(P_{3}+K_{1}\right) \vee 2 K_{1}$, $\overline{3 K_{2}}, K_{3,3}$, or $\overline{2 P_{3}}$. By Theorem 12 there are no minimal probe non- $\mathcal{P}(\mathcal{T})$ graphs that are trees. If $\bar{H}$ is the complement of a tree then, by Theorem $11 \mathrm{H}$ is $\overline{P_{6}}$, or $\overline{2 P_{3}}$, or $\overline{3 K_{2}}$. 
Lemma 26 Let $H$ be a spider with spider partition $(S, C, R)$. Then, $H$ is a probe $\mathcal{T}$ graph if and only if $H=$ thin $_{2}\left(t K_{1}\right)$ for some $t \geq 0$. Moreover, if $H$ is a probe $\mathcal{T}$ graph and $H^{\prime}$ is a fat spider that arises from $H$, then $H^{\prime}$ is also a probe $\mathcal{T}$ graph except when $t \geq 1$ and $H^{\prime}$ arises from $H$ by making a false twin of a vertex of $C$.

Proof: Let $H$ be a spider with partition $(S, C, R)$. Since $H$ is $\left(P_{4}+K_{1}\right)$-free and tent-free, $|C|=2$. Notice that $R$ is an independent set because $\operatorname{thin}_{2}\left(K_{2}\right)$ is a non- $\mathcal{P}(\mathcal{T})$ graph. We conclude that $H=$ $\operatorname{thin}_{2}\left(t K_{1}\right)$ for some $t \geq 0$. Clearly, $\operatorname{thin}_{2}\left(t K_{1}\right)$ is a probe $\mathcal{T}$ graph. By setting all vertices of $S \cup C$ as probe vertices $(P)$ and the vertices of $R$ as nonprobe vertices $(N)$ and adding all the edges whose endpoints belong to $N(F)$, we obtain the completion $H^{*}=(N \cup P, E(H) \cup F)$ of $H$ that is a $\mathcal{T}$-graph. Therefore, $H$ is a $\mathcal{P}(\mathcal{T})$-graph.

Suppose that $H$ is a $\mathcal{P}(\mathcal{T})$-graph and let $H^{\prime}$ be a fat spider arising from $H$. Let $v$ a false twin of a vertex $s \in S$. Consider the following probe partition $(N, P): N=R \cup\{v, s\}$ and $P=V(H)-N$ and denote by $F$ the edges whose endpoints belong to $N$. Consequently, the completion $H^{*}=(N \cup P, E(H) \cup F)$ is $\mathcal{T}$-graph. Now, let $v$ be a true twin of a vertex $s \in S$. Consider the following $(N, P)$ probe partition: $N=R \cup(S-\{s, v\})$ and $P=V(H)-N$. So, the completion $H^{*}=(V(H), E(H) \cup F)$ with probe partition $(N, P)$, where $F$ are the edges whose endpoints belong to $N$, is a $\mathcal{T}$-graph. Therefore, if $H^{\prime}$ arises by making a twin of a vertex $s \in S$, then $H^{\prime}$ is a $\mathcal{P}(\mathcal{T})$-graph. We have already seen a probe interval partition of $H$ where each $c \in C$ is a probe vertex having a probe $\mathcal{T}$ completion. Therefore, if $H^{\prime}$ arises by making a true twin of a vertex of $C, H^{\prime}$ is also a $\mathcal{P}(\mathcal{T})$-graph. Finally, assume that $H^{\prime}$ arises by making a false twin of a vertex $c \in C$. If $t=0$, then $H^{\prime}$ is clearly a $\mathcal{P}(\mathcal{T})$-graph. If $t \geq 1$, then $H^{\prime}$ is not a probe $\mathcal{P}(\mathcal{T})$-graph because it contains an induced $C_{4}+K_{1}$.

Lemma 27 The minimally non- $\mathcal{P}(\mathcal{T})$ graphs that are spiders or fat spiders are tent and thin $n_{2}\left(K_{2}\right)$.

Proof: By minimality, $|C| \leq 3$ (otherwise contains $P_{4}+K_{1}$ or tent as proper induced subgraphs). If $|C|=3$, then $H$ is a thick spider (otherwise it contains $P_{4}+K_{1}$ as proper induced subgraph). If $|C|=3$ and $H$ is thick, then $H$ contains an induced tent and, by minimality, $H=$ tent. Therefore, we can assume that $|C|=2$. If $H[R]$ were a stable set then, by the above lemma, $H$ is a spider, a contradiction. Therefore, $R$ is not a stable set. So, $H$ contains an induced thin ${ }_{2}\left(K_{2}\right)$ and, by minimality, $H=\operatorname{thin}_{2}\left(K_{2}\right)$.

Suppose, by way of contradiction, that there is a fat spider $H^{\prime}$ that is a minimally non- $\mathcal{P}(\mathcal{T})$ graph. By minimality, $H^{\prime}$ arises from a spider $H$ that is a $\mathcal{P}(\mathcal{T})$-free graph. So, by the above lemma, $H=$ $\operatorname{thin}_{2}\left(t K_{1}\right)$ for some $t \geq 1$ and $H$ arises by making a false twin of a vertex of $C$. Then, $H^{\prime}$ contains an induced $C_{4}+K_{1}$. By minimality, $H^{\prime}=C_{4}+K_{1}$. This leads to a contradiction because $H^{\prime}$ is a fat spider. This contradiction proves that there are no minimally non- $\mathcal{P}(\mathcal{T})$ graphs that are fat spiders.

By combining Lemmas 23, 24, and 27, the following characterization is obtained.

Theorem 28 Let $G$ be a $P_{4}$-tidy graph. Then, $G$ is a $\mathcal{P}(\mathcal{T})$-graph if and only if $G$ contains no induced $2 K_{2}+K_{1}, P_{4}+K_{1}, C_{4}+K_{1},\left(K_{2}+2 K_{1}\right) \vee 2 K_{1},\left(P_{3}+K_{1}\right) \vee 2 K_{1}, \overline{3 K_{2}}, K_{3,3}, \overline{2 P_{3}}, C_{5}$, tent, or $\operatorname{thin}_{2}\left(K_{2}\right)$.

Proof: Let $H$ be a minimally non- $\mathcal{P}(\mathcal{T})$ graph that is a $P_{4}$-tidy graph. If $H$ is disconnected, then $H$ is isomorphic to either $2 K_{2}+K_{1}$, or $P_{4}+K_{1}$, or $C_{4}+K_{1}$. If $\bar{H}$ is disconnected, then $H$ is isomorphic to either $\left(K_{2}+2 K_{1}\right) \vee 2 K_{1}$, or $\left(P_{3}+K_{1}\right) \vee 2 K_{1}$, or $\overline{3 K_{2}}$, or $K_{3,3}$, or $\overline{2 P_{3}}$. If $H$ were $C_{5}, P_{5}$, or $\overline{P_{5}}$, 
then $H$ would be necessarily isomorphic to $C_{5}$. Finally, if $H$ is a spider or a fat spider, then $H$ is tent or $\operatorname{thin}_{2}\left(K_{2}\right)$.

\section{Partial characterizations of probe unit interval graphs}

To the best of our knowledge, the problem of finding all minimally non-(probe unit interval) graphs whose complement is disconnected remains open. Nevertheless, we solve this problem for the classes of treecographs and $P_{4}$-tidy graphs.

Lemma 29 The minimally non-(probe unit interval) graphs that are tree-cographs or $P_{4}$-tidy and whose complement is disconnected are $\left(2 K_{2}+K_{1}\right) \vee K_{1},\left(P_{4}+K_{1}\right) \vee K_{1},\left(C_{4}+K_{1}\right) \vee K_{1}$, thin $n_{2}\left(K_{2}\right) \vee K_{1}$, $\left(K_{2}+2 K_{1}\right) \vee 2 K_{1},\left(P_{3}+K_{1}\right) \vee 2 K_{1}, \overline{3 K_{2}}, K_{3,3}$, and $\overline{2 P_{3}}$.

Proof: The result follows from Theorem 22. Indeed, the companion of the class of unit interval graphs is the class $\mathcal{T}$, and: (i) the minimally non- $\mathcal{P}(\mathcal{T})$ graphs that are tree-cographs or $P_{4}$-tidy and are probe unit interval graphs are $2 K_{2}+K_{1}, P_{4}+K_{1}, C_{4}+K_{1}$, and $\operatorname{thin}_{2}\left(K_{2}\right)$ (see Theorem 25 and Theorem 28; ; (ii) by the proof of Lemma 24 , the minimal forbidden induced subgraphs of $\mathcal{P}(\mathcal{K}) \cup \mathcal{T}$ are $K_{2}+2 K_{1}$, $P_{3}+K_{1}, C_{4}$, and $C_{5}$ (all of which are probe unit interval except for $C_{5}$ ); and (iii) the only minimally non- $\mathcal{T}$ graph that is probe complete is $3 K_{1}$.

Theorem 30 Let $G$ be a tree-cograph. Then, $G$ is a probe unit interval graph if and only if $G$ contains no induced bipartite-claw, $L, H_{n}$ for any $n \geq 1, \overline{P_{6}},\left(2 K_{2}+K_{1}\right) \vee K_{1},\left(P_{4}+K_{1}\right) \vee K_{1},\left(C_{4}+K_{1}\right) \vee K_{1}$, $\left(K_{2}+2 K_{1}\right) \vee 2 K_{1},\left(P_{3}+K_{1}\right) \vee 2 K_{1}, \overline{3 K_{2}}, K_{3,3}$, or $\overline{2 P_{3}}$.

Proof: Let $H$ be a minimally non-(probe unit interval) graph that is a tree-cograph. By minimality, $H$ is connected. If $H$ is a tree, then, by Theorem 5, $H$ is bipartite-claw, $L$, or $H_{n}$ for some $n \geq 1$. If $H$ is the complement of a tree, then, by Theorem 11, $H$ is $\overline{P_{6}}$. If $\bar{H}$ is disconnected, by Lemma 29, $H$ is $\left(2 K_{2}+K_{1}\right) \vee K_{1},\left(P_{4}+K_{1}\right) \vee K_{1},\left(C_{4}+K_{1}\right) \vee K_{1},\left(K_{2}+2 K_{1}\right) \vee 2 K_{1},\left(P_{3}+K_{1}\right) \vee 2 K_{1}, \overline{3 K_{2}}, K_{3,3}$, $\overline{2 P_{3}}$ (because thin ${ }_{2}\left(K_{2}\right) \vee K_{1}$ is not a tree-cograph).

Lemma 31 Let $H$ be a spider with spider partition $(S, C, R)$. Then, $H$ is a probe unit interval if and only if $|C|=2$ and $H[R]$ is probe complete. Moreover, if $H$ is probe unit interval and $H^{\prime}$ is a fat spider that arises from $H$, then $H^{\prime}$ is also probe unit interval except when $H[R]$ is not a complete graph and $H^{\prime}$ arises by making a false twin of a vertex of $C$.

Proof: Let $H$ be a probe unit interval spider with spider partition $(S, C, R)$. Notice that $|C|=2$ because otherwise $H$ would contain either an induced net or an induced tent. In addition, $H[R]$ is $\left\{\overline{P_{3}}, C_{4}\right\}$-free (otherwise, $H$ contains an induced $\operatorname{thin}_{2}\left(\overline{P_{3}}\right)$ which is not a probe unit interval graph or $\left.\left(C_{4}+K_{1}\right) \vee K_{1}\right)$. So, $H[R]$ is probe complete. Conversely, if $H[R]$ is probe complete and $|C|=2$, clearly $H$ is a probe unit interval graph.

Suppose now that $H$ is probe unit interval. The vertices of $S$ in any probe interval partition of $H$ can be probe or nonprobe, so if $H^{\prime}$ arises by making a twin of a vertex of $S$, then $H^{\prime}$ is also a probe unit interval graph. The vertices of $C$ in any probe interval partition of $H$ can be set as probe, so if $H^{\prime}$ arises by making a true twin of $C, H^{\prime}$ is also probe unit interval. Finally, suppose that $H^{\prime}$ arises from $H$ by 
making a false twin of $C$. If $H[R]$ is a complete graph, then $H^{\prime}$ is clearly probe interval, but if $H[R]$ is not a complete graph, $H^{\prime}$ contains an induced $\left(P_{3}+K_{1}\right) \vee 2 K_{1}$.

Lemma 32 The minimally non-(probe unit interval) graphs that are spiders or fat spiders are net, tent, and $\operatorname{thin}_{2}\left(\overline{P_{3}}\right)$.

Proof: Let $H$ be a spider with spider partition $(S, C, R)$ that is a minimally non-(probe unit interval) graph. If $|C| \geq 3$, by minimality, $H$ is net or tent. So we may assume that $|C|=2$. Since $H$ is not a probe unit interval graph, $H[R]$ is non-(probe complete). So, $H[R]$ contains an induced $\overline{P_{3}}$ or $C_{4}$. If $H[R]$ contained an induced $C_{4}, H$ would contain an induced $\left(C_{4}+K_{1}\right) \vee K_{1}$ and, by minimality, $H=\left(C_{4}+K_{1}\right) \vee K_{1}$, contradicting the fact that $H$ is a spider. So, necessarily $H[R]$ contains an induced $\overline{P_{3}}$. Therefore, $H$ contains an induced $\operatorname{thin}_{2}\left(\overline{P_{3}}\right)$ and, by minimality, $H=\operatorname{thin}_{2}\left(\overline{P_{3}}\right)$.

Suppose, by way of contradiction, that there is a fat spider $H^{\prime}$ that is a minimally non-(probe unit interval). By the minimality and the above lemma, $H^{\prime}$ arises from a spider $H$ with $|C|=2$ and $H[R]$ probe complete by making a false twin of a vertex of $C$. But then, $H^{\prime}$ contains an induced $\left(C_{4}+K_{1}\right) \vee K_{1}$ and, by minimality, $H^{\prime}=\left(C_{4}+K_{1}\right) \vee K_{1}$, contradicting the fact that $H^{\prime}$ is a fat spider.

Theorem 33 Let $G$ be a $P_{4}$-tidy graph. Then, $G$ is a probe unit interval graph if and only if $G$ contains no induced $\left(2 K_{2}+K_{1}\right) \vee K_{1},\left(P_{4}+K_{1}\right) \vee K_{1},\left(C_{4}+K_{1}\right) \vee K_{1}$, thin ${ }_{2}\left(K_{2}\right) \vee K_{1},\left(K_{2}+2 K_{1}\right) \vee 2 K_{1}$, $\left(P_{3}+K_{1}\right) \vee 2 K_{1}, \overline{3 K_{2}}, K_{3,3}, \overline{2 P_{3}}, C_{5}$, net, tent, or thin ${ }_{2}\left(\overline{P_{3}}\right)$.

Proof: Let $H$ be a minimally non-(probe unit interval) that is $P_{4}$-tidy. By minimality, $H$ is connected. If $\bar{H}$ is disconnected, by Lemma 29 is $\left(2 K_{2}+K_{1}\right) \vee K_{1},\left(P_{4}+K_{1}\right) \vee K_{1},\left(C_{4}+K_{1}\right) \vee K_{1}$, $\operatorname{thin}_{2}\left(K_{2}\right) \vee K_{1},\left(K_{2}+2 K_{1}\right) \vee 2 \overline{K_{1}},\left(P_{3}+K_{1}\right) \vee 2 K_{1}, \overline{3 K_{2}}, K_{3,3}$, or $\overline{2 P_{3}}$. If $H$ were $C_{5}, P_{5}$, or $\overline{P_{5}}$, necessarily $H=C_{5}$. If $H$ is a spider or a fat spider, then $H$ is net, tent, or $\operatorname{thin}_{2}\left(\overline{P_{3}}\right)$.

\section{Acknowledgments}

The authors would like to thank the anonymous referees for their comments and suggestions.

\section{References}

[1] D. Bayer, V. Bang Le, and H. N. de Ridder. Probe threshold and probe trivially perfect graphs. Theoretical Computer Science, 410(47-49):4812-4822, 2009.

[2] D. E. Brown and J. R. Ludgren. Bipartite probe interval graphs, circular-arc graphs, and interval point bigraphs. Australasian Journal of Combinatorics, 35:221-236, 2006.

[3] D. E. Brown, J. R. Ludgren, and L. Sheng. Characterization of cycle-free unit probe interval graphs. Discrete Applied Mathematics, 157:762-767, 2009.

[4] D. Chandler, D.-M. Chang, T. Kloks, J. Liu, and S.-L. Peng. On probe permutation graphs. Discrete Applied Mathematics, 157:2611-2619, 2009.

[5] D. Chandler, M.-S. Chang, T. Klocks, J. Liu, and S.-L. Peng. Recognition of probe and partitioned probe distance hereditary graphs. Lecture Notes in Computer Science, 4041:267-278, 2006. 
[6] D. Chandler, M.-S. Chang, T. Kloks, V. Bang Le, and S.-L. Peng. Probe Ptolemaic graphs. In COCOON, pages 468-477, 2008.

[7] V. Chvátal and P. L. Hammer. Aggregation of inequalities in integer programming. Technical Report CS-TR-75-518, Stanford University, 1975.

[8] V. Giakoumakis, F. Roussel, and H. Thuillier. On $P_{4}$-tidy graphs. Discrete Mathematics \& Theoretical Computer Science, 1(1):17-41, 1997.

[9] M. C. Golumbic and M. Lipshteyn. Chordal probe graphs. Discrete Applied Mathematics, 143(13):221-237, 2004.

[10] M. C. Golumbic and A. N. Trenk. Tolerance Graphs. Cambridge University Press, New York, 2004.

[11] C. Hoàng. Perfect graphs. PhD thesis, School of Computer Science, McGill University, Montreal, 1985.

[12] B. Jamison and S. Olariu. A new class of brittle graphs. Studies in Applied Mathematics, 1203:8992, 1989.

[13] B. Jamison and S. Olariu. On a unique tree representation for $P_{4}$-extendible graphs. Discrete Applied Mathematics, 34:151-164, 1991.

[14] V. B. Le and H. N. de Ridder. Probe split graphs. Discrete Mathematics \& Theoretical Computer Science, 9(1), 2007.

[15] C. Lekkerkerker and D. Boland. Representation of finite graphs by a set of intervals on the real line. Fundamenta Mathematicae, 51:45-64, 1962.

[16] J. Liu and H. Zhou. Dominating subgraphs in graphs with some forbidden structures. Discrete Mathematics, 135:163-168, 1994.

[17] N. Pržulj and D. Corneil. 2-tree probe interval grahps have a large obstruction set. Discrete Applied Mathematics, 150:216-231, 2005.

[18] F. Roberts. Indifference graphs. In F. Harary, editor, Proof Techniques in Graph Theory, pages 139-146. Academic Press, 1969.

[19] L. Sheng. Cycle-free probe interval graphs. Congressus Numerantium, 140:33-42, 1999.

[20] G. Tinhofer. Strong tree-cographs are Birkhoff graphs. Discrete Applied Mathematics, 22(3):275288,1989 .

[21] G. Wegner. Eigenschaften der Nerven homologisch-einfacher Familien in $\mathbb{R}^{n} . \mathrm{PhD}$ thesis, Göttingen, 1967.

[22] D. B. West. Introduction to Graph Theory. Prentice Hall, New Jersey, USA, second edition, 2001.

[23] P. Zhang, P. Schon, E. Fischer, E. Gayanis, J. Weiss, S. Wistler, and P. Bourne. An algorithm based on graph theory for the assembly of contigs in physical mapping of DNA. Bioinformatics, 10(3):309-317, 1994. 